\title{
Influence des redoux dans la genèse des grandes crues alpines : cas de la dernière crue d'inondation de l'Isère à Grenoble en
} 1859

\section{The influence of warmer weather periods on high river episodes in the French Alps: the case of the last flood of the Isère River in Grenoble in 1859}

\section{Dominique Dumas}

Volume 24, numéro 2, 2011

Reçu le 19 octobre 2009, accepté le 7 juillet 2010

URI : https://id.erudit.org/iderudit/1006108ar

DOI : https://doi.org/10.7202/1006108ar

Aller au sommaire du numéro

Éditeur(s)

Université du Québec - INRS-Eau, Terre et Environnement (INRS-ETE)

ISSN

0992-7158 (imprimé)

1718-8598 (numérique)

Découvrir la revue

Citer cet article

Dumas, D. (2011). Influence des redoux dans la genèse des grandes crues alpines : cas de la dernière crue d'inondation de l'Isère à Grenoble en 1859. Revue des sciences de l'eau / Journal of Water Science, 24(2), 147-158.

https://doi.org/10.7202/1006108ar

\section{Résumé de l'article}

L'inondation de 1859 marque la dernière grande crue d'inondation de l'Isère. La tranquillité de l'Isère au cours du $\mathrm{XX}^{\mathrm{e}}$ siècle est-elle définitive, est-elle liée à une modification climatique? Pour répondre à ces questions, l'étude vise dans un premier temps à cerner les particularités climatiques de ces événements hydrologiques. Dans un second temps, afin d'évaluer le caractère plus ou moins exceptionnel de ces conditions climatiques, les valeurs annuelles maximales des précipitations cumulées, d'une journée à six journées consécutives, ont été ajustées à la loi de Gumbel. La démarche a ensuite été reconduite avec les précipitations journalières couplées à une hausse des températures. Il apparaît d'une manière assez nette que ces crues font suite à des conditions climatiques véritablement exceptionnelles; un épisode pluvieux durable qui se combine avec une phase de redoux. Aussi, l'absence de ces grandes crues au cours $\mathrm{XX}^{\mathrm{e}}$ siècle n'est pas étonnante sur un plan statistique, et il ne faudrait peut-être pas y voir la marque d'un changement climatique régional ou planétaire.
Tous droits réservés @ Revue des sciences de l'eau,
Ce document est protégé par la loi sur le droit d'auteur. L'utilisation des services d'Érudit (y compris la reproduction) est assujettie à sa politique d'utilisation que vous pouvez consulter en ligne. 


\section{INFLUENCE DES REDOUX DANS LA GENĖSE DES GRANDES CRUES ALPINES : CAS DE LA DERNIÈRE CRUE D'INONDATION DE L'ISÈRE À GRENOBLE EN 1859}

The influence of warmer weather periods on high river episodes in the French Alps: the case of the last flood of the Isère River in Grenoble in 1859

DOMINIQUe DUMAS

Université Joseph-Fourier, Institut de Géographie Alpine

14 bis, avenue Marie-Reynoard, 38100 Grenoble, France

Reçu le 19 octobre 2009, accepté le 7 juillet 2010

\section{RÉSUMÉ}

L'inondation de 1859 marque la dernière grande crue d'inondation de l'Isère. La tranquillité de l'Isère au cours du $\mathrm{XX}^{\mathrm{e}}$ siècle est-elle définitive, est-elle liée à une modification climatique? Pour répondre à ces questions, l'étude vise dans un premier temps à cerner les particularités climatiques de ces événements hydrologiques. Dans un second temps, afin d'évaluer le caractère plus ou moins exceptionnel de ces conditions climatiques, les valeurs annuelles maximales des précipitations cumulées, d'une journée à six journées consécutives, ont été ajustées à la loi de Gumbel. La démarche a ensuite été reconduite avec les précipitations journalières couplées à une hausse des températures. Il apparaît d'une manière assez nette que ces crues font suite à des conditions climatiques véritablement exceptionnelles; un épisode pluvieux durable qui se combine avec une phase de redoux. Aussi, l'absence de ces grandes crues au cours $\mathrm{XX}^{\mathrm{e}}$ siècle n'est pas étonnante sur un plan statistique, et il ne faudrait peut-être pas y voir la marque d'un changement climatique régional ou planétaire.

Mots-clés : Pluies, températures, crues exceptionnelles, changement climatique, l'Isère, Grenoble.
ABSTRACT

The great 1859 flood at Grenoble in the southeastern France was the last water-level rise of such magnitude for the Isère River, and Grenoble has not been flooded again since that time. However, one can legitimately wonder whether the calm behavior of the Isère during the 20th century was something definitive or whether it was related to climate change. The present study aimed at answering this question, firstly by determining the climatic characteristics of the hydrological episodes in question, while, secondly, using Gumbel's Law to adjust the maximum annualvalues of accumulated precipitation over a period lasting from one to six consecutive days, in order to assess how exceptional such climatic conditions actually are. The procedure was then repeated by linking daily precipitation to rises in temperature. It is clearly shown that flood episodes follow truly exceptional climatic conditions. Thus, the absence of such severe floods during the 20th century is not statistically surprising, and perhaps should not be seen as a sign of regional or planetary climate change.

Keywords: Rain, temperature, exceptional water-level rise, climate change, Isère River, Grenoble. 


\section{INTRODUCTION}

Tout au long de l'histoire, les inondations de l'Isère à Grenoble ont été nombreuses. Parmi les grandes crues de l'Isère, celles successives du 16 puis du 30 novembre 1651 semblent être les plus importantes. Un peu plus de deux cents ans après, l'inondation de 1859 marque la dernière grande crue d'inondation de l'Isère (COSSERON DE VILLENOISY, 1894; DUMAS, 2004; EPTEAU, 1996; PARDÉ, 1925a). Depuis, la ville de Grenoble n'a non seulement plus jamais été inondée, et les crues les plus puissantes ont à peine dépassé la moitié du débit maximal enregistré en 1859 (Figure 1). Certes, les caractéristiques du bassin et du lit de l'Isère ont été fortement modifiées depuis la seconde moitié du XIX ${ }^{\mathrm{e}}$ siècle, notamment par des travaux d'endiguement. Par ailleurs, la forte dynamique géomorphologique de ce cours d'eau alpin est également à prendre en compte, puisqu' elle a fortement modifié les caractéristiques morphométriques de son lit (PEIRY, 1997; VAUTIER, 2000). Pour autant, il serait sans doute insensé de croire que l'Isère est définitivement réduite à l'état d'un cours d'eau inoffensif et " il faut s'attendre un jour ou l'autre à l'inondation presque totale de Grenoble et du Grésivaudan » (PARDÉ, 1937).

À partir de divers documents et d'archives (Fonds Pardé, Fonds Dauphinois, Archives Départementales de l'Isère), il est possible de retrouver des informations concernant le contexte climatique de ces événements hydrologiques extrêmes, et tout particulièrement de la dernière grande crue de l'Isère en 1859. La caractérisation, même imparfaite, des conditions climatiques de cette inondation permet ensuite de confronter cette connaissance à une approche probabiliste du contexte climatique susceptible d'avoir favorisé l'apparition de cet événement. Il est en effet certain que des pluies importantes, cumulées sur plusieurs jours, sont à l'origine de la crue de 1859. Mais étaient-elles véritablement exceptionnelles? Cet événement n'est-il pas aussi, et peut-être surtout, la conséquence d'une conjonction inattendue de conditions pluviales et thermiques? Le bassin versant de la moyenne Isère est très largement montagneux, aussi des précipitations durables associées à une phase de redoux, et donc accompagnant une remontée en altitude de l'isotherme $0{ }^{\circ} \mathrm{C}$, peuvent favoriser un écoulement exceptionnel. D’un côté, la rétention nivale des précipitations est alors plus réduite, et de l'autre, l'écoulement est accru par une alimentation complémentaire, consécutive à une fusion nivale du manteau neigeux.

À partir de séries pluviométriques et thermiques de plusieurs postes de mesure, cette étude vise à mieux cerner l'importance probable de conditions pluvio-thermiques exceptionnelles de la dernière grande inondation de Grenoble, et précise ainsi un peu mieux les conditions climatiques de cet événement.

\section{SAISONNALITÉ DES GRANDES CRUES DE L'ISÈRE}

La distribution annuelle des crues de l'Isère sont souvent présentées (PARDÉ, 1925a; PUPIER, 1996; VAUTIER, 2000; VIVIAN, 1969) avec une opposition entre le printemps et l'automne caractéristique des régimes des rivières alpines (PARDÉ, 1964). Pour l'Isère, l'occurrence mensuelle des crues peut être tout de même précisée (Figure 2). Les crues les plus nombreuses surviennent au début de la saison chaude en raison principalement des débits de base gonflés par la fusion nivale. Lors de ces événements, l'alimentation nivale des crues reste possible en juin, voire juillet, et elle demeure néanmoins probablement assez modeste dans la majorité des cas. Sur cette période de hautes eaux, il suffit de précipitations abondantes, mais pas nécessairement exceptionnelles, pour générer le débit maximal de l’année.

En revanche, les grandes crues, que nous définissons ici comme supérieures à $900 \mathrm{~m}^{3} \bullet \mathrm{s}^{-1}$, apparaissent essentiellement en novembre (Figure 2), sans pour autant que les précipitations soient plus abondantes sur cette période de l'année (PUPIER, 1996). Aussi, il n'est pas impossible que les plus grandes crues connues de l'Isère soient souvent d'origine nivo-pluviale, car c'est précisément à ce moment de l'année que des pluies abondantes peuvent être facilement associées à une remontée des températures efficace vis-à-vis de l'écoulement. Il ne fait pas alors encore suffisamment froid pour qu'un redoux ne puisse plus favoriser des précipitations liquides sur une

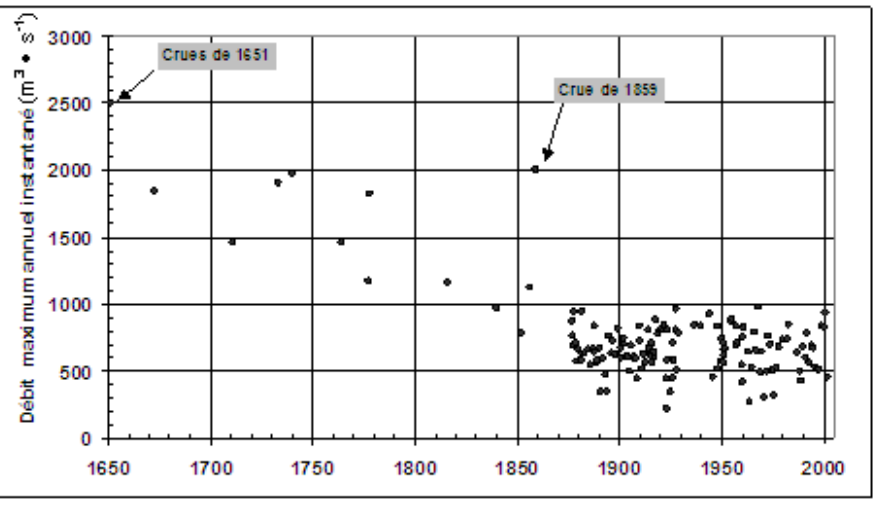

Figure 1. Évolution des crues annuelles depuis 1651 jusqu’à 2002. Données annuelles continues de 1877 à 2002. Données : PARDÉ (1925a, 1925b), VIVIAN (1969), VIVIAN et al. (1987), documents des Ponts et Chaussées figurant dans le fonds Pardé, de documents fournis par la DDE Isère, et données DIREN.

The evolution of annual high water levels, 1651 - 2002. Continuous annual data from 1877 to 2002. Sources: PARDÉ (1925a, 1925b), VIVIAN (1969), VIVIAN et al. (1987), Ponts et Chaussées [Road Works Department] documents included in the Fonds Pardé [Pardé Collection], documents provided by DDE Isère, and DIREN data. 


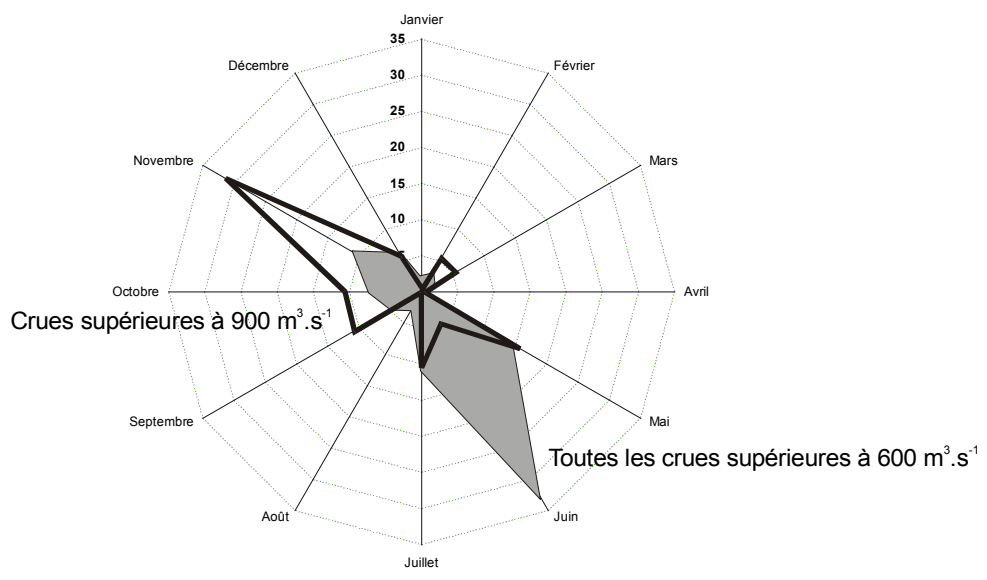

Figure 2. Distribution mensuelle des crues annuelles de l'Isère à Grenoble (en \%) sur la période 1651 à 2002 (à partir des données représentées sur la figure 1).

Monthly distribution of the Isère River annual high-water levels at Grenoble (in \%) from 1651 to 2002 (based on data shown in Fig. 1).

majeure partie du bassin. Et à l'inverse, les températures sont alors suffisamment basses pour qu'un manteau neigeux puisse s'installer. Dès lors, l'influence nivale directe (fusion) ou indirecte (faible rétention des pluies sous forme neigeuse), guidée par une hausse des températures, ne serait peut-être pas à négliger pour comprendre les grandes crises hydrologiques de l'Isère.

\section{LES CONDITIONS CLIMATIQUES LORS DE L'INONDATION DE 1859}

À partir de différents fonds et de sources bibliographiques, plusieurs paramètres climatiques associés aux grandes crues de l'Isère ont pu être renseignés (GUETNY, 2004). La lecture attentive de diverses sources (Fonds Anciens Dauphinois : dossiers 26784, 26789, 26800,26798; Fonds Pardé : dossiers 1091, 1155; BERTRAND, 1859; CHAMPION, 1861; COSSERON DE VILLENOISY, 1894; MARMILLOD, 1778; PILOT, 1857; PILOT, 1859; PILOT DE THOREY, 1765; RAULLIN, 1876; VIDAL, 1904), il a été possible de mieux cerner, non seulement les précipitations responsables de ces crues, mais également d'obtenir des indications sur les caractéristiques thermiques qui ont accompagné certains de ces événements hydrologiques. Par exemple, l'épisode pluviométrique de septembre 1733 est décrit précisément par MARCHAND (1876) comme " un ouragan " qui débuta le 14 septembre vers $16 \mathrm{~h}$ et les principales rivières « sortirent de leurs lits dès $18 \mathrm{~h}$ ». Lintensité de l'averse ne semble pas faire de doute et la fusion nivale ne peut expliquer cette réponse hydrologique aussi rapide, d'autant que les précipitations mesurées à Grenoble, relevées en une journée, auraient été supérieures à $160 \mathrm{~mm}$ (5 pieds)! En revanche, la crue du 27 octobre 1778, associée au " Déluge de la Saint-Crépin », est consécutive à une période très pluvieuse associée à un épisode de redoux (MARMILLOD, 1778). En 36 heures, des pluies "diluviennes " s'abattirent sur la région accompagnées d'une forte hausse des températures : une température de $16{ }^{\circ} \mathrm{C}$ est relevée à Grenoble. Des observations très précises de la crue de 1859 figurent dans l'ouvrage de PILOT (1859). L'auteur note ainsi une température de $18-19^{\circ} \mathrm{C}$ à Grenoble, et évoque des précipitations continues sur Grenoble du 31 octobre au 2 novembre, précédées de pluies le 26 et le 29 octobre. Récemment, deux séries de températures journalières relevées au centre-ville de Grenoble ont été découvertes (AUFFRAY et al., 2009). L'une publiée dans les «Bulletins de la Société de statistique, des sciences naturelles et des arts industriels du département de l'Isère " (Figure 3), et l'autre dans un relevé personnel effectué de 1852 à 1863 par Joseph-Eustache Flandrin. Dans la première série, les précipitations sont également précisées et quantifiées; on retrouve, par exemple, $54 \mathrm{~mm}$ de pluie cumulée du 29 octobre au 31 octobre, avec des précipitations nulles le 30 octobre. Dans la seconde série, en revanche, une description qualitative, généralement sous la forme " pluie " ou " soleil ", est simplement indiquée. Du 29 octobre au $1^{\text {er }}$ novembre 1859 , des précipitations y sont mentionnées, comme le décrit d'ailleurs PILOT (1859). Les deux séries mentionnent les températures relevées le matin (ou la température minimale), à midi, et l'après-midi (ou la température maximale). Dans les deux cas, une température maximale de $20^{\circ} \mathrm{C}$ est relevée le $1^{\text {er }}$ novembre 1859 à Grenoble. Les relevés ne sont cependant pas toujours strictement identiques, même s'ils montrent une évolution générale des températures relativement similaire. La hausse des températures moyennes journalières, le $1^{\text {er }}$ novembre 1859 , serait ainsi de $4^{\circ} \mathrm{C}$ ou $6{ }^{\circ} \mathrm{C}$ selon la série. 


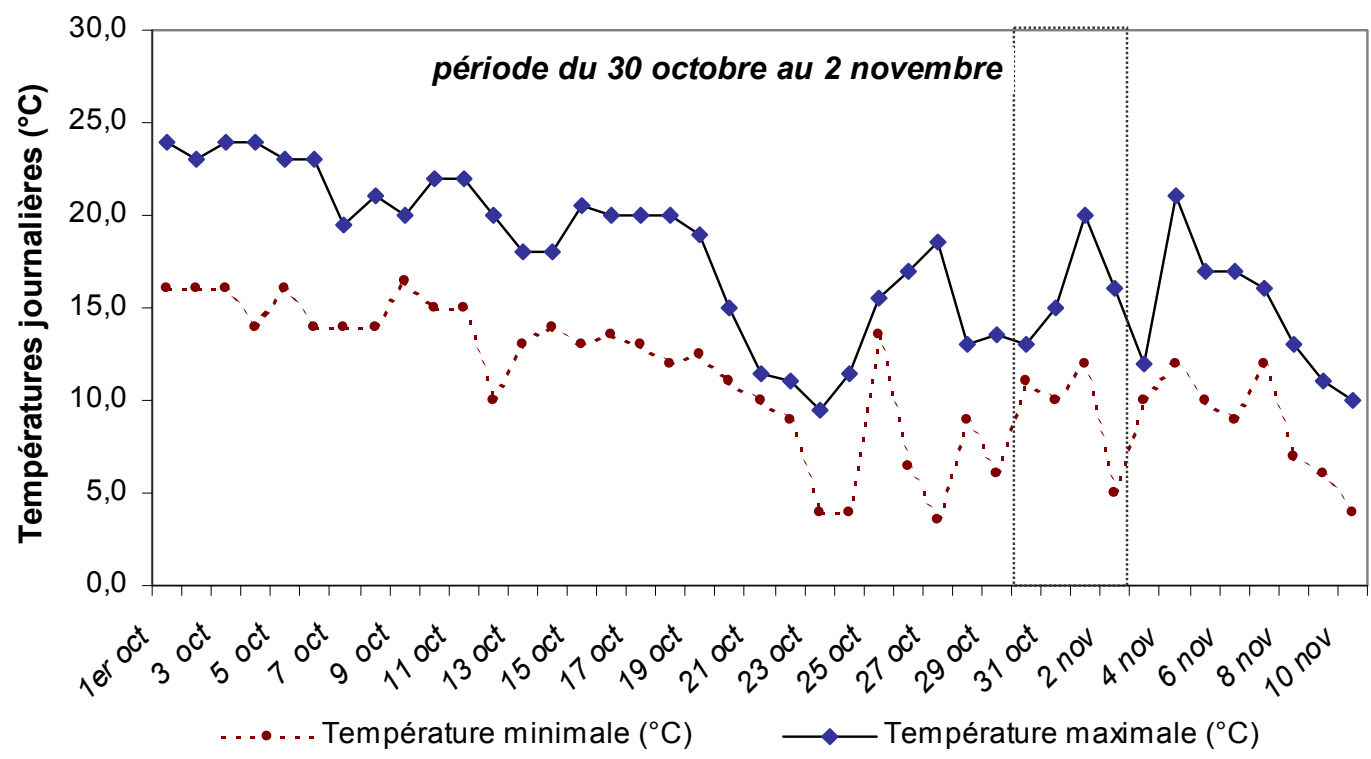

Figure 3. Évolution de la température journalière maximale et minimale en octobre et novembre 1859. Observations à Grenoble centre-ville, effectuées par L. Demarchi, Archives Météo-France CDM38, dans AUFFRAY et al., 2009 (correction des températures minimales le 31 octobre et le 3 novembre). Evolution of maximum and minimum daily temperatures in October and November 1859. Recorded in Grenoble city centre by Demarchi, Archives Météo-France CDM38, in AUFFRAY et al., 2009 (minimum temperatures corrected for 31 October and 3 November).

C’est aussi à partir des observations de PILOT (1859) que PARDÉ (Figure 4) a reconstruit sommairement un hydrogramme de cette crue, avec un pic positionné à $1800 \mathrm{~m}^{3} \bullet \mathrm{s}^{-1}$ (DUMAS, 2004). L'analyse de ce document a été conduite par PARDÉ (Figure 4) directement sur le document (Figure 4). Sa démarche repose alors sur la nécessité d'effectuer les calculs à la main (on procéderait probablement autrement aujourd'hui), et vise en premier lieu à cerner l'ordre de grandeur des volumes écoulés et des précipitations totales responsables de cette crue.

Ainsi, il calcule le volume total écoulé en additionnant successivement, du 30 octobre au 8 novembre, le débit moyen journalier (déterminé par le débit à $12 \mathrm{~h}$ ). Il chiffre ainsi à 5450 le cumul des débits moyens journaliers sur ces dix jours. Il estime le débit journalier de base sur cette période à $100 \mathrm{~m}^{3} \bullet \mathrm{s}^{-1}$ en moyenne (il note $\mathrm{d}=100$ ) et trouve ainsi un volume de crue de 388 millions $\mathrm{m}^{3}$ [(5450-10*100) $\left.3600 * 24\right]$, qu'il arrondit ensuite à 400 millions $\mathrm{m}^{3}$.

En se basant sur la température alors relevée à Grenoble (PILOT, 1859) et un gradient thermique d'environ $1{ }^{\circ} \mathrm{C} / 100 \mathrm{~m}$, il situe l'isotherme $0{ }^{\circ} \mathrm{C}$ tour à tour à $2000 \mathrm{~m}$ puis à $2200 \mathrm{~m}$ d'altitude. Dès lors, il suppose explicitement qu'une partie des précipitations a été neigeuse et n'a donc pas contribué directement à la crue. Il estime ainsi une lame d'eau écoulée comprise entre $100 \mathrm{~mm}$ (au-dessous de $2200 \mathrm{~m}$ la superficie du bassin est d'environ $4000 \mathrm{~km}^{2}$ ) et $113 \mathrm{~mm}$ (au-dessous de $2000 \mathrm{~m}$ la superficie contributive du bassin est de $3550 \mathrm{~km}^{2}$ ). Selon une démarche sensiblement analogue, il suppose que le coefficient d'écoulement est inscrit entre 0,6 et 0,75 . Les précipitations responsables de cette crue seraient donc situées entre 166 et $188 \mathrm{~mm}$, et entre 133 à $150 \mathrm{~mm}$ avec un coefficient d'écoulement plus fort de 0,75 (Tableau 1).

En période pluvieuse, où l'air est facilement saturée, le gradient proposé par PARDÉ (Figure 4) demeure quand même assez élevé, et probablement peu réaliste. Par temps humide et perturbé, le gradient moyen pour l'ensemble du bassin est alors vraisemblablement plus proche du gradient pseudoadiabatique (ou " adiabatique saturée») dont la valeur à $1000 \mathrm{hPa}$ est proche de $0,5{ }^{\circ} \mathrm{C} \cdot 100 \mathrm{~m}^{-1}$ pour une température de l'air de $15{ }^{\circ} \mathrm{C}$. Il augmente en revanche légèrement lorsque la température s'abaisse (TRIPLET et ROCHE, 1971). Une étude récente sur la reconstitution des conditions climatiques de cette crue de 1859 décrit une remontée brutale de l'isotherme $0{ }^{\circ} \mathrm{C}$, sur près 1600 m de dénivelé, du 30 octobre au $1^{\text {er }}$ novembre (OBLED et al., 2009). Selon les auteurs de cette étude, l'isotherme $0{ }^{\circ} \mathrm{C}$ serait alors portée à $3200 \mathrm{~m}$ d'altitude. Toutefois, la limite pluie-neige n'est pas strictement placée au niveau de $l^{\prime}$ isotherme $0{ }^{\circ} \mathrm{C}$. Généralement, on observe cette limite à $+2^{\circ} \mathrm{C}$, c'est-à-dire souvent à une altitude d'environ 300 à $500 \mathrm{~m}$ sous l'isotherme $0{ }^{\circ} \mathrm{C}$ (LHOTELLIER, 2005). Par ailleurs, cette étude ne vise pas à reconstituer strictement jour après jour l'ensemble du contexte pluviothermique de cette crue, mais à étudier cet événement dans sa globalité afin de tenter d'estimer ses possibilités de retour, et de décrire l'importance, notamment dans les zones montagneuses, de considérer également les phases de redoux lors de ces crises hydrologiques. Sur l'ensemble des jours pluvieux lors de la crue, la limite moyenne pluie-neige 


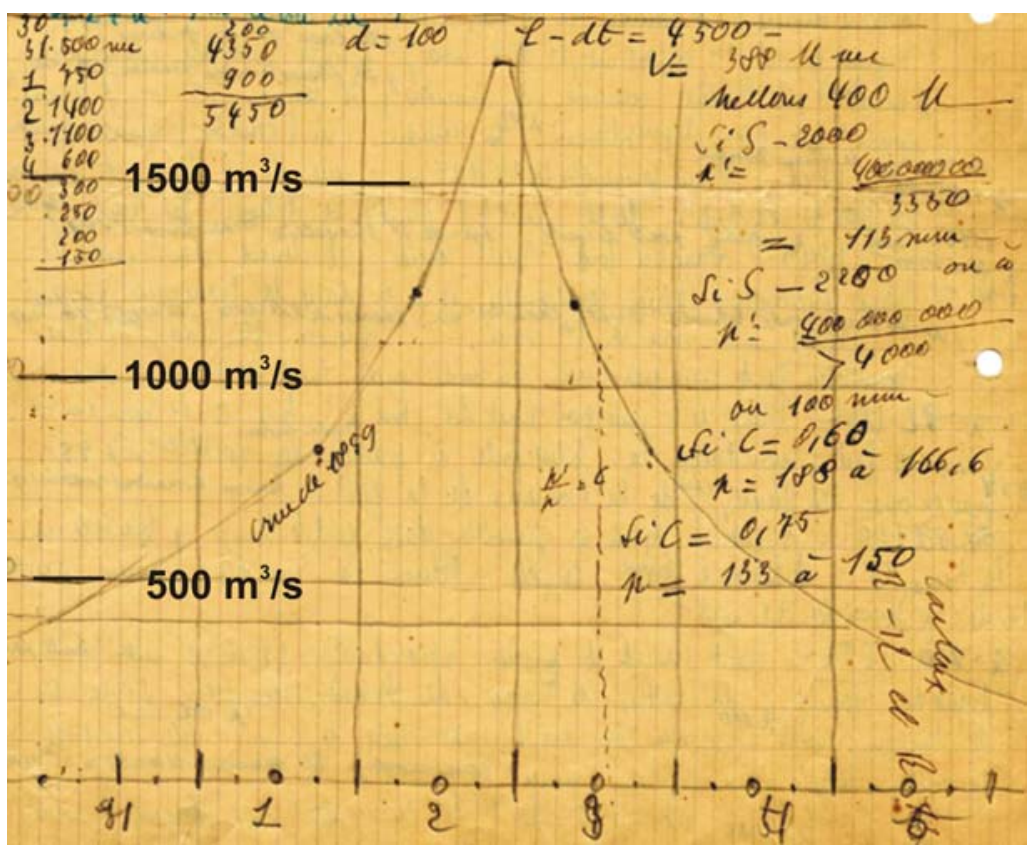

Figure 4. Hydrogramme de la crue de 1859 et calculs de M. Pardé (Fonds Pardé, dossier 1051).

Hydrogram of the 1859 flood, with calculations by Pardé (archive reference: Fonds Pardé, dossier 1051).

Tableau 1. Synthèse des hypothèses de M. Pardé, et nouvelles hypothèses. Estimation des précipitations totales lors de l'événement (en gras les valeurs les plus vraisemblables).

Table 1. Synthesis of Pardés hypotheses, plus new hypotheses. Estimation of total precipitation during the flood episode (the most likely values are shown in bold type).

\begin{tabular}{|c|c|c|c|c|c|c|}
\hline & \multirow[b]{2}{*}{$\begin{array}{c}\text { Altitude de la } \\
\text { limite pluie- } \\
\text { neige }(\mathrm{m})\end{array}$} & \multirow[b]{2}{*}{$\begin{array}{l}\text { Part du bassin } \\
\text { concerné par la } \\
\text { pluie (\%) }\end{array}$} & \multirow{2}{*}{$\begin{array}{c}\text { Surface du } \\
\text { bassin affectée } \\
\text { par la pluie } \\
\left(\mathrm{km}^{2}\right)\end{array}$} & \multirow[b]{2}{*}{$\begin{array}{l}\text { Lame d'eau } \\
\text { écoulée }(\mathrm{mm})\end{array}$} & \multicolumn{2}{|c|}{ Précipitations $(\mathrm{mm})$} \\
\hline & & & & & $\begin{array}{c}\text { Avec coef. } \\
\text { d'écoulement } \\
\text { de } 0,6\end{array}$ & $\begin{array}{c}\text { Avec coef. } \\
\text { d'écoulement } \\
\text { de } 0,75\end{array}$ \\
\hline \multirow{2}{*}{$\begin{array}{l}\text { Hypothèse de } \\
\text { M. Pardé }\left(^{*}\right)\end{array}$} & 2000 & $60,6 \%$ & 3469 & 115 & 192 & 154 \\
\hline & 2200 & $69,6 \%$ & 3979 & 101 & 168 & 134 \\
\hline \multirow{4}{*}{$\begin{array}{l}\text { Hypothèses } \\
\text { vraisemblables }\end{array}$} & 2300 & $74,1 \%$ & 4240 & 94 & 157 & 126 \\
\hline & 2500 & $82,8 \%$ & 4736 & 84 & 141 & 113 \\
\hline & 2700 & $89,7 \%$ & 5131 & 78 & 130 & 104 \\
\hline & 2900 & $94,8 \%$ & 5423 & 74 & 123 & 98 \\
\hline
\end{tabular}

$\left.{ }^{*}\right)$ En prenant en compte les véritables valeurs de surface du bassin pour les altitudes

${ }^{*}$ ) Taking into account the actual surface values according to altitude

se place sans doute plus vraisemblablement à $2300 \mathrm{~m}$, voire $2500 \mathrm{~m}$ d'altitude (Tableau 1). Pendant la phase de crue, elle correspond d'ailleurs assez bien à l'altitude reconstituée moyenne de l'isotherme $0{ }^{\circ} \mathrm{C}$ (OBLED et al., 2009).

Plusieurs épisodes pluvieux ont précédé l'événement (AUFFRAY et al., 2009; OBLED et al., 2009; PILOT, 1859), ce qui laisse penser que les sols étaient alors relativement saturés. Néanmoins, le coefficient d'écoulement moyen proposé par PARDÉ (Figure 4) de 0,75, apparaît très élevé.
Par exemple, même si le contexte et l'intensité sont différents, la crue de l'Isère en mars 2001, dont le volume de crue est d'environ 80 millions $\mathrm{m}^{3}$, est consécutive à des pluies de bassin de l'ordre de $100 \mathrm{~mm}$ (réanalyses SAFRAN), elle ne peut donc être associée à un coefficient d'écoulement aussi fort (le débit maximal enregistré à Grenoble est de $940 \mathrm{~m}^{3} \bullet \mathrm{s}^{-1}$ le 22 mars à $18 \mathrm{~h}$ ). Le coefficient d'écoulement, en reprenant l'hypothèse proposée par PARDÉ (Figure 4), se rapproche sans doute davantage de $60 \%$ que de $75 \%$. Les précipitations responsables de cette crue seraient donc vraisemblablement assez proches de 
140-160 mm, cumulées sur plusieurs jours (Tableau 1). Ces précipitations, cumulées sur deux ou trois jours, voire un peu plus, ont-elles été exceptionnelles?

\section{PROBABILITÉ DE RETOUR DE CET ÉPISODE PLUVIEUX}

Dans le cadre de cette étude, nous disposons des pluies journalières moyennes pour le bassin de l'Isère à Grenoble sur la période 1970 à 2006. Ces pluies de bassin sont issues des réanalyses pluviométriques SAFRAN, après réaggrégation des valeurs produites sur les mailles SAFRAN, et la prise en compte des limites du bassin à partir d'un modèle numérique de terrain à $1 \mathrm{~km}$ (BEN DAOUD et al., 2009; DURAND et al., 2009; SAUQUET, 2006; VIDAL et al., 2009).

Certes, un siècle environ sépare la dernière inondation de Grenoble du début de cette chronique pluviométrique, cependant, une étude des précipitations sur Grenoble montre qu'il n'existe pas véritablement de divergence dans le comportement pluviométrique des périodes 1886-1950 et 1950 à nos jours (PUPIER, 1996). Afin de cerner le caractère plus ou moins exceptionnel d'un cumul de pluie allant d'une journée à six jours consécutifs, nous avons tracé pour ces précipitations de bassin des courbes journalières intensité - durée - fréquence (Figure 5). Les valeurs annuelles maximales des précipitations cumulées, d'une journée à six journées consécutives, ont été ajustées à la loi de Gumbel afin d'en apprécier les hauteurs pour différentes fréquences ou périodes de retour. Cette loi, à deux paramètres, est en effet largement utilisée en France et dans différents contextes climatiques (BOUVARD et GARROS BERTHET, 1994; BUISHAND, 2008; DJERBOUA et al., 2004; GUILLOT et DUBAND, 1969; HERSHFIELD et KOHLER, 1960; KIEFFER et BOIS, 1997; KIEFFER WEISSE, 1998; KIEFFER WEISSE et BOIS 2001a, 2001b, 2002; MOSQUERA-MACHADO, 2007; WOTLING et al., 2000). Elle n'a cependant pas vocation universelle même si elle est souvent privilégiée dans les études hydroclimatiques. Il existe naturellement d'autres lois d'ajustement statistique d'extrêmes (BOIS et al., 1997; KIEFFER WEISSE, 1998), mais l'ajustement avec des lois à plus de deux paramètres n'est pas forcément toujours plus judicieux; pour obtenir des estimations robustes, il est souvent préférable d'utiliser des lois simples avec un minimum de paramètres (DJERBOUA, 2001).

Sur une journée, un épisode pluvieux supérieur à $150 \mathrm{~mm}$, comme ce fut peut-être le cas pour l'inondation de 1733, apparaît exceptionnel sur un plan statistique puisque la période de retour avoisinerait mille ans (Figure 5). En revanche, les précipitations consécutives et cumulées sur trois à cinq jours supérieures à $150 \mathrm{~mm}$, voire $160 \mathrm{~mm}$, ne sont pas rares (Figure 5). Sur trois jours consécutifs, par exemple, la valeur de $150 \mathrm{~mm}$ est dépassée pour une fréquence d'environ 0,95 ( $\mathrm{T}=20$ ans). Elle approcherait même une période de retour de cinq ans pour des pluies cumulées sur cinq jours consécutifs. Or, un épisode pluvieux s'échelonnant sur cette durée, tout au moins sur une partie du bassin, reste envisageable pour l'inondation de 1859 (PILOT, 1859).

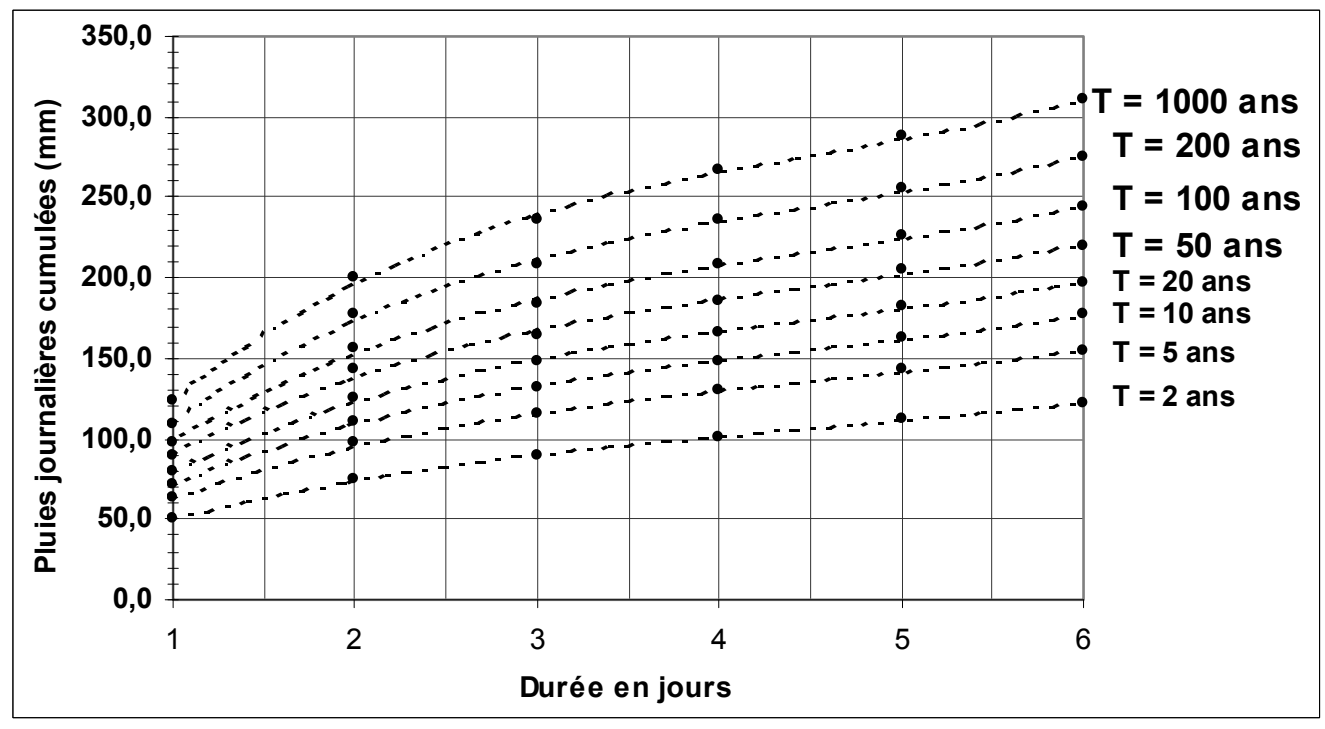

Figure 5. Courbes des hauteurs de pluie cumulées sur plusieurs jours consécutifs, durées, fréquences pour le bassin versant de l'Isère : période de référence 1970-2006. Données réanalyses pluviométriques SAFRAN (SAUQUET 2006; VIDAL et al., 2009). Accumulated rainfall over several consecutive days, duration and frequency for the Isère River basin: reference period 1970-2006. SAFRAN reanalysed rainfall data (SAUQUET 2006; VIDAL et al., 2009). 
Ceci montre bien, en considérant uniquement ce paramètre pluviométrique, le caractère, certes peu fréquent (vingtennal, ou à peine un peu plus), mais non exorbitant de précipitations susceptibles d'avoir engendré une crue comparable à celle de 1859. C'est donc peut-être la concomitance de précipitations durables et intenses avec une fusion nivale du manteau neigeux par situation de redoux, par exemple, qui serait à l'origine des événements les plus marquants. Ces précipitations, souvent cumulées sur plusieurs jours, associées ou non à une hausse de température, ont-elles été exceptionnelles?

\section{HAUSSE DES TEMPÉRATURES ASSOCIÉE À UN ÉPISODE PLUVIEUX EXCEPTIONNEL}

Afin d'apprécier l'occurrence d'événements pluvieux associés à une hausse des températures, de nouvelles courbes journalières intensité-durée-fréquence ont été établies sur les précipitations journalières cumulées associées à une hausse des températures. Afin d'évaluer l'intensité d'une remontée significative des températures, cette étude tente de s'appuyer sur des caractéristiques climatiques relevées lors des grandes crues de l'Isère. La lecture attentive de plusieurs sources bibliographiques décrivant les crues anciennes de l'Isère montre clairement que certaines grandes crues ont été concomitantes avec une hausse des températures, généralement observée à Grenoble. Un redoux, d'au moins $4^{\circ} \mathrm{C}$, est ainsi noté pour les crues de l'Isère le 11 février 1711, le 21 décembre 1740, le 2 juillet 1777 , le 27 octobre 1778 , le 31 mai 1856 et le 2 novembre 1859 (GUETNY, 2004). La précision des hausses de température, observées à partir de mesures instrumentales au cours du XVIII siècle, peut paraître suspecte. Cependant, différentes études conduites à partir d'observations des températures journalières relevées à Paris, dès 1676, ont montré qu'après étalonnage et conversion des valeurs en degrés Celsius, il était possible de suivre précisément l'évolution des températures journalières à Paris depuis cette date (DETTWILLER, 1981; LEGRAND et LE GOFF, 1992; ROUSSEAU, 2009).

Lors des crues anciennes de l'Isère, les hausses de température observées ont été généralement relevées à Grenoble. Certes, ces périodes de redoux appréciées ponctuellement ne caractérisent pas les conditions thermiques sur l'ensemble du bassin de l'Isère lors de l'événement. La connaissance des températures à l'échelle d'un bassin montagneux, et au pas de temps journalier, reste d'ailleurs toujours délicate, plus encore sur les valeurs extrêmes (GOTTARDI, 2009; LHOTELLIER, 2005). Sur l'ensemble des Alpes, LHOTELLIER (2005) réalise à partir de régressions multiples une spatialisation des températures, cohérente à l'échelle annuelle et mensuelle, mais nettement moins fiable à l'échelle journalière, où la spatialisation des températures est même jugée par l'auteur comme souvent "décevante ". Cependant, sans chercher à reconstituer les conditions thermiques sur l'ensemble du bassin, ces informations ponctuelles sur les hausses brutales de la température, retrouvées dans divers documents et archives, peuvent être facilement comparées à des mesures récentes de température relevées alors, elles aussi, aux alentours de Grenoble.

Afin d'évaluer les hausses de température sur la période 1970 à 2006, période pour laquelle nous possédons les précipitations journalières sur le bassin versant, la chronique des températures de la station du Versoud (Météo France), située à environ $10 \mathrm{~km}$ de Grenoble, a été retenue. Cette série couvre la période 1999 à 2006. En utilisant les enregistrements quotidiens de 1969 à 2001 de la station de St-Martin d'Hères (Météo France), située près de Grenoble, il a été possible d'étendre les observations de la station du Verrsoud. Cette reconstruction des températures journalières au Versoud de 1969 à 1999 est rendue envisageable par la relation élevée $\left(\mathrm{R}^{2}\right.$ supérieur à 0,99$)$ des enregistrements des deux stations sur la période commune d'observation allant du $1^{\text {er }}$ juin 1999 au 16 novembre 2001 (Figure 6).

Différentes méthodes ont été testées afin de détecter les phases de redoux à partir de la série d'observations mesurées et reconstituées de la station du Versoud. La différence entre la température moyenne journalière et la température journalière moyennée sur 27 jours (moyenne mobile centrée et calculée sur 27 jours) a été retenue pour faire ressortir les phases de redoux (Figure 7).

L'objectif n'est pas ici de repérer strictement des périodes chaudes (à partir d'un seuil thermique déterminé pour l'année, par exemple), mais bien de détecter une phase de redoux dans un contexte climatique saisonnier, qui est alors susceptible d'apporter soudainement un soutien supplémentaire à l'écoulement par fusion nivale. On suppose qu'un manteau neigeux est préexistant sur une partie du bassin versant. Pour conforter cette hypothèse, les phases de redoux associées à des pluies consécutives sont repérées uniquement les mois de l'année où un manteau neigeux peut être présent sur une partie significative du bassin versant. Les réanalyses pluviométriques SAFRAN fournissent pour le bassin de l'Isère les précipitations liquides et neigeuses sur la période 1970 à 2006 (Figure 8). Elles montrent que la part des précipitations neigeuses devient prépondérante de novembre à avril; le coefficient de nivosité moyen est alors supérieur à $50 \%$. Un apport supplémentaire dans l'écoulement lié à une fusion nivale ne semble donc que très difficilement pouvoir apparaître entre mai et octobre.

De 1970 à 2006, en retenant uniquement les mois de novembre à avril, il est ainsi possible de combiner les jours de pluie consécutifs associés exclusivement à une phase de redoux 


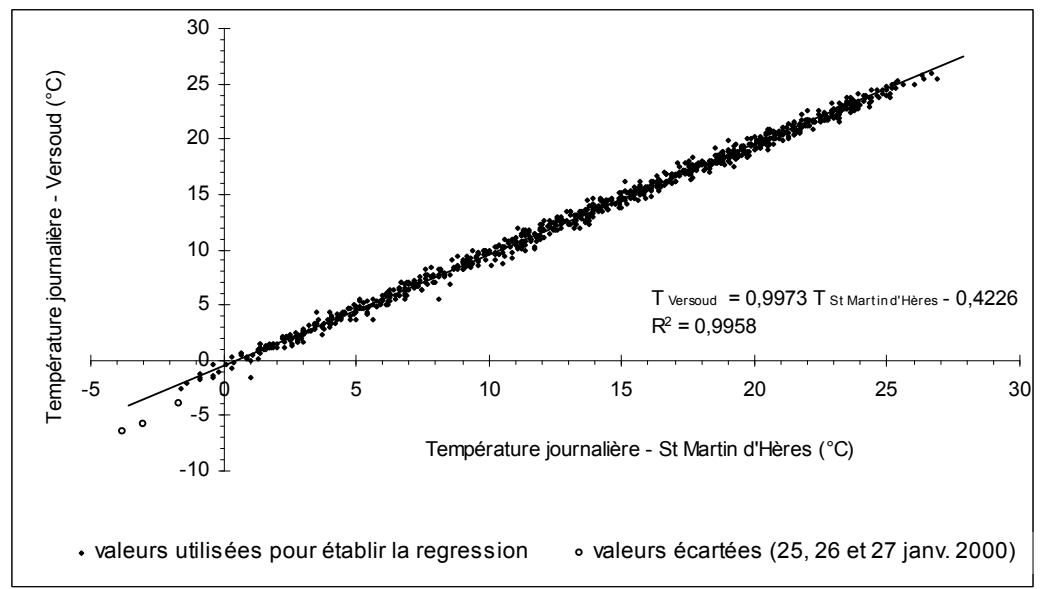

Figure 6. Relation entre les températures mesurées à St-Martin d'Hères et celles mesurées au Versoud sur la période commune du 1 juin 1999 au 16 novembre 2001.

The relationship between temperatures measured at St-Martin d'Hères and at Le Versoud over the same period from 1 June 1999 to 16 November 2001.

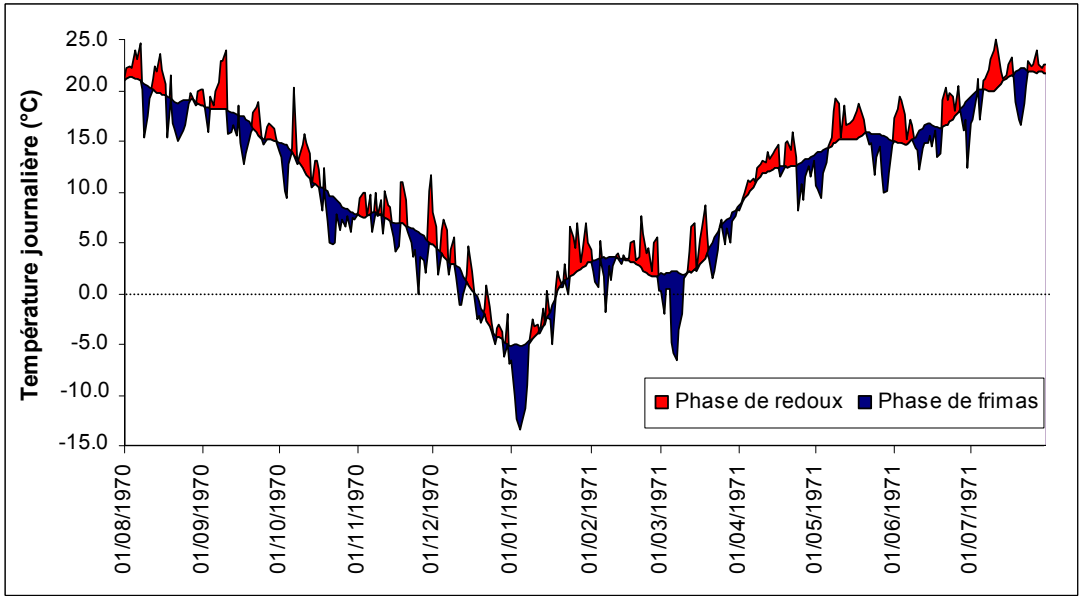

Figure 7. Évolution des températures journalières et évolution saisonnière calculée à partir des moyennes mobiles sur 27 jours, à la station du Versoud : exemple avec les années 1970 et 1971.

The evolution of daily temperatures and seasonal evolution calculated from sliding means over 27 days at Le Versoud weather station: an example based on years 1970 and 1971.

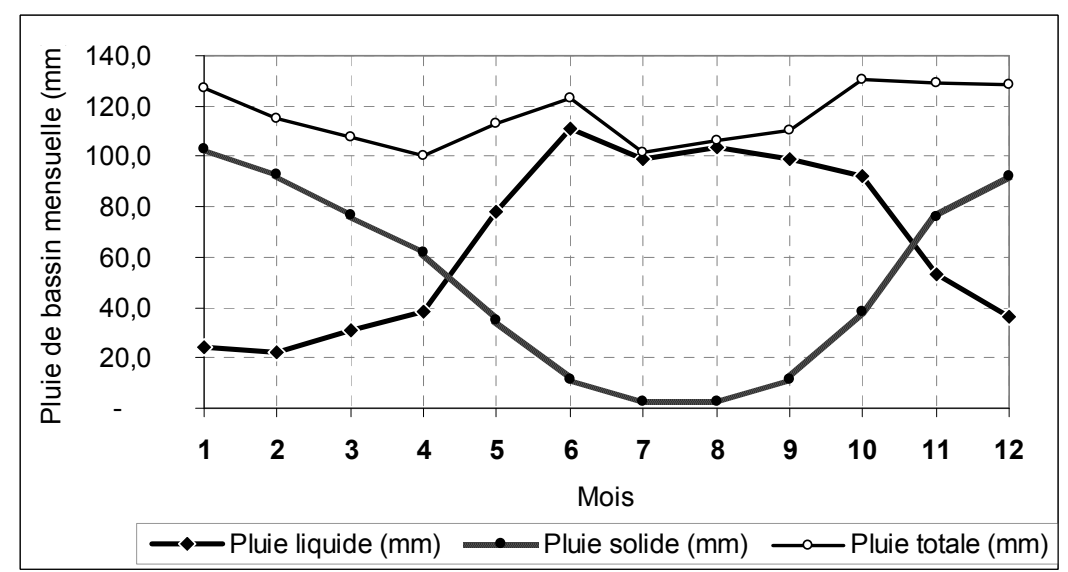

Figure 8. Nature des précipitations mensuelles moyennes pour le bassin de l'Isère à Grenoble : période 1970 à 2006 (réanalyses pluviométriques SAFRAN). Type of mean monthly precipitation values (rain, snow, total) for the Isère River basin at Grenoble, 1970 - 2006 (SAFRAN rainfall reanalysis). 
qui intègre au moins une journée où la hausse de température est supérieure à $4{ }^{\circ} \mathrm{C}$. Ce seuil de $4{ }^{\circ} \mathrm{C}$ a été retenu afin de rejoindre les conditions de redoux observées lors de la crue de 1859, et des crues anciennes de l'Isère (Figure 9). Cette phase de redoux est définie par une différence positive entre la température moyenne journalière et la température obtenue à partir d'une moyenne mobile centrée, calculée sur 27 jours. Pour chaque année, la valeur maximale des pluies consécutives (cumulées sur $\mathrm{N}$ jours) lors d'une phase de redoux, avec une hausse d'au moins $4{ }^{\circ} \mathrm{C}$ sur une journée, a été retenue. Cependant, lorsque, pour une année, cette valeur maximale des pluies pour $\mathrm{N}$ jours consécutifs est inférieure aux pluies calculées pour $\mathrm{N}-1$ jours, la valeur la plus grande est conservée afin de garder une croissance monotone des courbes. Un ajustement par la loi de Gumbel est ensuite réalisé sur ces valeurs maximales annuelles s'échelonnant de 1970 à 2006.

Le résultat montre que des précipitations cumulées, supérieures à $150 \mathrm{~mm}$, associées à une phase de redoux, deviennent plus rares. Pour un cumul de $160 \mathrm{~mm}$ sur deux ou trois jours, les fréquences redeviennent plus réalistes (proches de la fréquence centennale), et montrent qu'il est sans doute utile, pour mieux définir les possibilités de retour de certains événements hydrologiques, de prendre en compte, avec les précipitations, les conditions thermiques. La crue de 1859 , où les précipitations ont été associées à un redoux, semble ainsi témoigner de caractéristiques pluviothermiques qui seraient alors plus proches d'une fréquence centennale si on considère, par exemple, des pluies cumulées sur trois jours supérieures à $160 \mathrm{~mm}$.

Dès lors, on peut imaginer qu'une concomitance de plusieurs facteurs soit nécessaire pour expliquer et comprendre les grandes crues de l'Isère, dont la dernière remonte à 150 ans. La puissance et l'extension de l'épisode pluvieux restent naturellement la clé pour déclencher ces crises hydrologiques, mais elles ne semblent pas suffisantes.

\section{CONCLUSION.}

Les grandes crues de l'Isère à Grenoble, supérieures à $1000 \mathrm{~m}^{3} \bullet \mathrm{s}^{-1}$, observées entre le XVI ${ }^{\mathrm{e}}$ et XIX ${ }^{\mathrm{e}}$ siècle, restent dès événements exceptionnels dont l'origine est sans doute le plus souvent à rechercher dans une concomitance de facteurs climatiques. Bien évidemment, les précipitations doivent être importantes, généralement durables, mais il ne semble pas qu'elles soient nécessairement exceptionnelles si l'on regarde les enregistrements pluviométriques fournis par plusieurs stations climatiques de la région, et par les réanalyses pluviométriques SAFRAN. Lorsque l'on regarde les épisodes pluvieux associés à une hausse des températures, les probabilités d'occurrence de ces événements redeviennent plus réalistes au regard des crises hydrologiques passées de l'Isère. Les précipitations durables associées à un redoux, même modéré, restent en matière de

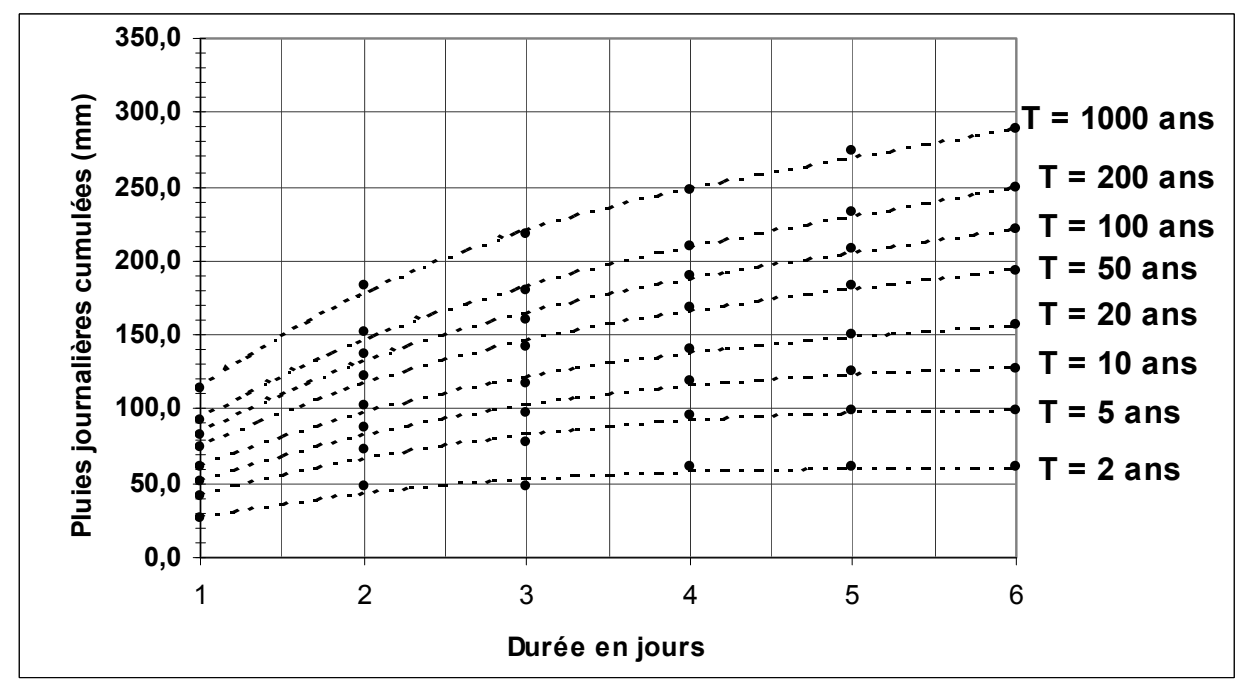

Figure 9. Courbes durées, fréquences des hauteurs de pluie cumulées sur plusieurs jours consécutifs associées à un redoux et intégrant une hausse journalière d'au moins $4^{\circ} \mathrm{C}$ observées à Grenoble pour le bassin versant de l'Isère : période de référence 1970-2006. Données : réanalyses pluviométriques SAFRAN, températures relevées au Versoud (Météo France).

Duration and frequency of accumulated rainfall over several consecutive days associated with subsequent warmer weather including a daily rise of at least $4^{\circ} \mathrm{C}$ measured at Grenoble for the Isère River basin. Reference period 1970 - 2006. Data sources: SAFRAN rainfall reanalysis, temperatures measured at Le Versoud (Météo France). 
probabilité plus rares. Indirectement, ces probabilités laissent donc entrevoir un rôle non négligeable des températures dans la genèse de ces grandes crues. Elles montrent aussi la nécessité de ne pas considérer exclusivement les pluies dans les estimations des événements hydrologiques rares, et peut-être, notamment en zone de montagne où la fusion nivale peut devenir non négligeable, de prendre avec précaution les valeurs des débits extrêmes estimées à partir des pluies comme, par exemple, la méthode du Gradex (BOUVARD et GARROS BERTHET, 1994; GUILLOT et DUBAND, 1969). Cette méthode est extrêmement astucieuse, efficace et robuste et, dans la majorité des cas, elle n'intègre cependant pas, si les conditions d'enneigement $\mathrm{du}$ bassin le permettent, l'amplification possible de l'écoulement par une hausse concomitante de la température avec l'épisode pluvieux. Enfin, cette analyse des données climatiques, couplée à une information historique sur les grandes crues, montre sans doute que la relative tranquillité de l'Isère au cours du $\mathrm{XX}^{\mathrm{e}}$ siècle serait plus liée à une carence d'événements climatiques, conjuguant de fortes précipitations et une situation de redoux, qu'une modification plus profonde des occurrences liée à une modification climatique régionale.

\section{REMERCIEMENTS}

L'auteur remercie Eric Sauquet (CEMAGREF, Lyon) pour lui avoir communiqué les réanalyses pluviométriques SAFRAN du bassin de l'Isère à Grenoble, et les relecteurs anonymes pour leurs remarques qui ont contribué à améliorer ce manuscrit.

\section{RÉFÉRENCES BIBLIOGRAPHIQUES}

AUFFRAY A., A. CLAVEL et S. JOURDAIN (2009). Contexte météorologique des mois d'octobre et novembre 1859. Dans : Colloque Crue Isère 1859-2009, 5 novembre 2009, Grenoble, France, 15 p.

BEN DAOUD A., E. SAUQUET et C. OBLED (2009). Reconstitution du risque nivo-pluviométrique durant le mois d'octobre 1859 par une technique d'analogie à l'aide des situations des années 1953-2005. Dans : Colloque Crue Isère 1859-2009, 5 novembre 2009, Grenoble, France, $25 \mathrm{p}$.

BERTRAND M.C. (1859). Étude sur l'inondation de Grenoble du 2 novembre 1859. Grenoble, $16 \mathrm{p}$.
BOIS P., C. OBLED, M.-F. SAINTIGNON et H. MAILLOUX (1997). Atlas expérimental des risques de pluies intenses : Cévennes, Vivarais. Pôle Grenoblois d'études et de recherche pour la prévention des risques naturels. INPG, UJF, CNRS, Grenoble, 19 planches, notice $4 \mathrm{p}$.

BOUVARD M. et H. GARROS BERTHET (1994). Les crues de projet des barrages : Méthode du GRADEX. Dans : Bulletin du comité français des grands barrages, $18^{\circ}$ congrès $C I G B / I C O L D, \mathrm{n}^{\circ} 2,94 \mathrm{p}$.

BUISHAND T.A. (2008). Statistics of extremes in climatology. Stat. Neerlandica, 43, 1-30.

CHAMPION M. (1861). Les inondations en France depuis le $V I^{e}$ siècle jusqu'à nos jours : recherches et documents contenant les relations contemporaines, les actes administratifs, les pièces officielles, etc. de toutes les époques. Paris, Cemagref 2000, 6 volumes.

COSSERON DE VILLENOISY M. (1894). Les inondations de l'Isère et du Drac. FAD, bibliothèque scientifique du Dauphiné, $22 \mathrm{p}$.

DETTWILLER J. (1981). Les températures annuelles à Paris durant les 300 dernières années. Météorologie, $6^{\mathrm{e}}$ série, 25, 103-109.

DJERBOUA A. (2001). Prédétermination des pluies et crues extrêmes dans les Alpes franco-italiennes: prévision quantitative des pluies journalières par la méthode des analogues. Thèse d'université, INPG (Institut polytechnique de Grenoble), Grenoble, France, 240 p.

DJERBOUA, A., D. DUBAND et P. BOIS (2004). Estimation des lois des précipitations extrêmes à partir de données journalières complètes. Houille Blanc., 3, 65-74.

DUMAS D. (2004). Les deux crues mémorables de l'Isère à Grenoble (1651 et 1859) : Analyse des estimations de M. Pardé. The two memorable floods on the Isère in Grenoble (1651 and 1859): An analysis of estimates by M. Pardé. Rev. Géogr. Alp., J. Alp. Res., 3, 27-49.

DURAND Y., M. LATERNSER, G. GIRAUD, P. ETCHEVERS, B. LESAFFRE et L. M'ERINDOL (2009). Reanalysis of 44 years of climate in the French Alps (1958-2002): methodology, model validation, climatology and trends for air temperature and precipitation. J. Appl. Meteor. Clim., 48, 429-449, DOI: 10.1175/2008JAMC1808.1. 
EPTEAU (S.A.) (1996). Étude d'impact des travaux d'aménagement de l'Isère en amont de Grenoble. A.D. IsèreDrac-Romanche, Rapport d'études, 230 p.

GOTTARDI F. (2009). Estimation statistique et réanalyse des précipitations en montagne. Utilisation d'ébauches par types de temps et assimilation de données d'enneigement. Application aux grands massifs montagneux français. Thèse d'université, INPG, LTHE-INPG (Laboratoire des Transferts en Hydrologie et Environnement - Institut polytechnique de Grenoble), Grenoble, France, 284 p.

GUETNY T. (2004). Les origines météorologiques des grandes crues de l'Isère à Grenoble depuis 1651. Mémoire de master première année. Institut de Géographie Alpine, Univ. Joseph Fourier, Grenoble, France, 134 p.

GUILLOT M.P. et D. DUBAND (1969). La methode du gradex pour le calcul de la probabilité des crues à partir des pluies. Int. Ass. Hydrolog. Sci., 84, 560-569.

HERSHFIELD D.M. et M.A. KOHLER (1960). An empirical appraisal of the Gumbel extreme value procedure. $J$. Geophys. Res., 65, 1737-1746.

KIEFFER A. et P. BOIS (1997). Variabilité des caractéristiques statistiques des pluies extrêmes dans les Alpes francaises. Rev. Sci. Eau, 10, 199-216.

KIEFFER WEISSE A. (1998). Étude des précipitations exceptionnelles de pas de temps court en relief accidenté (Alpes françaises). Méthode de cartographie des précipitations extrêmes. Relations avec le contexte topographique. Utilisation de l'information au pas de temps de la journée. Thèse ???, INPG, LTHE - INPG (Laboratoire des Transferts en Hydrologie et Environnement - Institut polytechnique de Grenoble), Grenoble, France, 314 p.

KIEFFER WEISSE A. et P. BOIS (2001a). Estimation de paramètres statistiques des précipitations extrêmes dans les Alpes françaises. Houille Blanc., 1, 62-70.

KIEFFER WEISSE A. et P. BOIS (2001b). Topographic effects on statistical characteristics of heavy rainfall and mapping in the French Alps. Appl. Meteor. 40, 720-740.

KIEFFER WEISSE A. et P. BOIS (2002). A comparison of methods for mapping statistical characteristics of heavy rainfall in the French Alps: the use of daily information. Hydrol. Sci., J. Sci. Hydrol., 47, 739-752.

LEGRAND J.-P. et M. LE GOFF (1992). Les observations météorologiques de Louis Morin. Monographie $\mathrm{n}^{\circ}$ 6, Direction de la Météorologie nationale, Paris, France, 36 p.
LHOTELLIER R. (2005). Spatialisation des températures en zone de montagne alpine. Thèse de géographie, Univ. Joseph Fourier, Grenoble, France, 352 p.

MARCHAND F. (1876). Relation d'un déluge arrivé à Grenoble le 15/09/1733 - propos rapporté par M. Guigonnet. Bulletin de la société statistique du département de l'Isère, tome 16, 269-276.

MARMILLOD M. (1778). Observations et mémoires sur la crue de l'Isère du 27 octobre 1778 et sur l'endiguement de l'Isère. FAD (Fonds des Archives départementales de l'Isère), 21 p.

MOSQUERA-MACHADO S.A. (2007). Flood hazard assessment of Atrato River in Colombia. Water Resour. Manage., 21, 591-609.

OBLED C., G. PANTHOU, R. GARÇON et F. GOTTARDI (2009). Crue de l'Isère 1859 : reconstitution d'un scénario de précipitations et de températures pour la période $1^{\text {er }}$ ctobre - 5 novembre 1859. Dans : Colloque Crue Isère 1859-2009, 5 novembre 2009, Grenoble, France, 22 p.

PARDÉ M. (1925a). Le régime du Rhône. Étude hydrologique. Première partie, Étude générale, 887 p. Deuxième partie, La genèse des crues, 441 p., Univ. de Lyon, Institut des Études Rhodaniennes, Lyon, France.

PARDÉ M. (1925b). Calcul des débits du Rhône et de ses affluents. BIBLIOTHEQQUE DE L'INSTITUT DE GÉOGRAPHIE ALPINE (Éditeur), Université de Grenoble, Grenoble, France, 168 p.

PARDÉ M. (1937). Le Grésivaudan inondé : juin 1937. Rev. Géogr. Alp., XXV, 521-524.

PARDÉ M. (1964). Fleuves et rivières. $4^{\mathrm{e}}$ édition, coll. Armand Colin, $\mathrm{n}^{\circ} 155,223 \mathrm{p}$.

PEIRY J.L. (1997). Recherches en géomorphologie fluviale dans les hydrosystèmes fluviaux des Alpes du Nord. Habilitation à diriger les recherches. Univ. Joseph Fourier, Grenoble, 305 p.

PILOT DE THOREY E. (1765). Linondation de 1764 à Grenoble. FAD (Fonds des Archives départementales de l'Isère), Microfilms du Dauphiné Libéré, tome 32, 250 260-282.

PILOT J. (1857). Recherches sur les inondations dans la vallée de l'Isère de 1219 à nos jours. MAISONVILLE (Éditeurs), FAD (Fonds des Archives départementales de l'Isère), Grenoble, France, 144 p. 
PILOT J.-J.A. (1859). Grenoble inondé. $2^{\mathrm{e}}$ édition, MAISONVILLE et FILS et JOURDAN (Éditeurs), Grenoble, France, 112 p.

PUPIER N. (1996). Analyse des fluctuations récentes de la nappe d'un hydrosystème perturbé. L'Isère dans le Grésivaudan. Thèse de Géographie, Univ. Jospeh Fourier, Grenoble, France, 219 p.

RAULLINV. (1876). Observations pluviométriques faites dans la France de 1704 à 1870. Belles Lettres et Arts de Bordeaux, France.

ROUSSEAU D. (2009). Les températures mensuelles en région parisienne de 1676 à 2008. Météorologie, 67, 43-55.

SAUQUET E. (2006). Mapping mean annual river discharges: geostatistical developments for incorporating river network dependencies. J. Hydrol., 331, 300-314.

TRIPLET J.P. et G. ROCHE (1971). Météorologie générale. École Nationale de la Météorologie, Toulouse, France, $317 \mathrm{p}$.

VAUTIER F. (2000). Dynamique géomorphologique et végétalisation des cours d'eau endigués : l'exemple de l'Isère dans le Grésivaudan. Thèse de Géographie, Univ. Jospeh Fourier, Grenoble, France, 377 p.

VIDAL J.-P., E. MARTIN, L. FRANCHISTÉGUY, M. BAILLON et J.-M. SOUBEYROUX (2009). A 50-year high-resolution atmospheric reanalysis over France with the Safran system. Int. J. Clim., 30, 1627-1644.

VIDAL L. (1904). Sur la température et les précipitations à Grenoble et à la Tronche. FAD (Fonds des Archives départementales de l'Isère), Annales de l'Université de Grenoble, 8 p.

VIVIAN H. (1969). Les crues de l'Isère à Grenoble et l'aménagement actuel des digues. Rev. Geogr. Alp., LVIII, 53-84.

VIVIAN H., J. BARRET et R. NICOLLE (1987). L'aménagement de l'Isère. État de la protection de l'agglomération grenobloise contre les crues (1968-1987). Rev. Geogr. Alp., 3, 233-248.

WOTLING G., C. BOUVIER, J. DANLOUX et J.M. FRITSCH (2000). Regionalization of extreme precipitation distribution using the principal components of the topographical environment. J. Hydrol., 233, 86-101. 